\title{
Betti Numbers of Semialgebraic Sets Defined by Quantifier-Free Formulae*
}

\author{
Andrei Gabrielov ${ }^{1}$ and Nicolai Vorobjov ${ }^{2}$ \\ ${ }^{1}$ Department of Mathematics, Purdue University, \\ West Lafayette, IN 47907, USA \\ agabriel@math.purdue.edu \\ ${ }^{2}$ Department of Computer Science, University of Bath, \\ Bath BA2 7AY, England \\ nnv@cs.bath.ac.uk
}

\begin{abstract}
Let $X$ be a semialgebraic set in $\mathbb{R}^{n}$ defined by a Boolean combination of atomic formulae of the kind $h * 0$ where $* \in\{>, \geq,=\}, \operatorname{deg}(h)<d$, and the number of distinct polynomials $h$ is $k$. We prove that the sum of Betti numbers of $X$ is less than $O\left(k^{2} d\right)^{n}$.
\end{abstract}

Let an algebraic set $X \subset \mathbb{R}^{n}$ be defined by polynomial equations of degrees less than $d$. The well-known results of Oleinik, Petrovskii [8], [9], Milnor [6], and Thom [12] provide the upper bound

$$
\mathrm{b}(X) \leq d(2 d-1)^{n-1}
$$

for the sum of Betti numbers $\mathrm{b}(X)$ of $X$ (with respect to the singular homology). In a more general case of a set $X$ defined by a system of $k$ non-strict polynomial inequalities of degrees less than $d$, the sum of Betti numbers does not exceed $O(k d)^{n}$.

These results were later extended and refined. Basu [1] proved that if a semialgebraic set $X$ is basic (i.e., $X$ is defined by a system of equations and strict inequalities), or is defined by a Boolean combination (with no negations) of only non-strict or of only strict inequalities, then

$$
\mathrm{b}(X) \leq O(k d)^{n}
$$

* The first author was supported by NSF Grant \# DMS-0070666 and by the James S. McDonnell Foundation. The second author was supported by the European RTN Network RAAG 2002-2006 (Contract HPRN-CT2001-00271). 
where $k$ is the number of distinct polynomials in the defining formula (this is a relaxed form of Basu's bound, for a more precise description see [1], [2].) Papers [7] and [13] imply that if $X$ is compact and is defined by an arbitrary Boolean combination of equations or inequalities, then

$$
\mathrm{b}(X) \leq O(k d)^{2 n} .
$$

The purpose of this note is to prove a bound for an arbitrary semialgebraic set defined by an arbitrary Boolean formula. More precisely, let $X$ be a semialgebraic set in $\mathbb{R}^{n}$ defined by a Boolean combination of atomic formulae of the kind $h * 0$ where $* \in\{>$, $\geq,=\}, \operatorname{deg}(h)<d$, and the number of distinct polynomials $h$ is $k$.

Theorem 1. The sum of Betti numbers of $X$ is less than $O\left(k^{2} d\right)^{n}$.

We deduce Theorem 1 from the following result.

Proposition 2 [1]. Let the Boolean combination which defines $X$ contain only nonstrict inequalities and no negations. Then the sum of Betti numbers of $X$ is less than $O(k d)^{n}$.

Since sums of Betti numbers of sets $X$ and $X \cap\left\{x_{1}^{2}+\cdots+x_{n}^{2}<\Omega\right\}$ coincide for a large enough $\Omega \in \mathbb{R}$ (see Lemma 1 of [1]), we assume in what follows that $X$ is bounded.

Definition 3. For a given finite set $\left\{h_{1}, \ldots, h_{k}\right\}$ of polynomials $h_{i}$ define its $\left(h_{1}, \ldots\right.$, $h_{k}$ )-cell (or just cell) as a semialgebraic set in $\mathbb{R}^{n}$ of the kind

$$
\left\{h_{i_{1}}=\cdots=h_{i_{k_{1}}}=0, h_{i_{k_{1}+1}}>0, \ldots, h_{i_{k_{2}}}>0, h_{i_{k_{2}+1}}<0, \ldots, h_{i_{k}}<0\right\},
$$

where $i_{1}, \ldots, i_{k_{1}}, \ldots, i_{k_{2}}, \ldots, i_{k}$ is a permutation of $1, \ldots, k$.

Obviously, for a given set of polynomials any two distinct cells are disjoint. According to [4] and [5], the number of all non-empty $\left(h_{1}, \ldots, h_{k}\right)$-cells is at most $(k d)^{O(n)}$, but

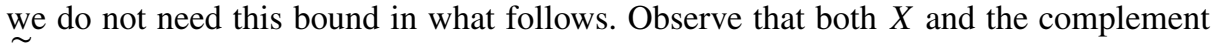
$\widetilde{X}=\mathbb{R}^{n} \backslash X$ are disjoint unions of some non-empty $\left(h_{1}, \ldots, h_{k}\right)$-cells.

Example 4. Let $X:=\left\{(x, y) \in \mathbb{R}^{2} \mid x^{2} y^{2}>0 \vee x^{2}+y^{2}=0\right\}$, i.e., $X$ is the plane $\mathbb{R}^{2}$ minus the union of the coordinate axes plus the origin. There are nine $\left(x^{2} y^{2}, x^{2}+y^{2}\right)$-cells among which exactly three,

$$
\left\{x^{2} y^{2}=x^{2}+y^{2}=0\right\},\left\{x^{2} y^{2}>0, x^{2}+y^{2}>0\right\} \text {, and }\left\{x^{2} y^{2}=0, x^{2}+y^{2}>0\right\},
$$

are non-empty. The union of the first two of these cells is $X$.

Introduce the following partial order on the set of all cells. Let $\Gamma \prec \Gamma^{\prime}$ iff the cell $\Gamma^{\prime}$ is obtained from the cell $\Gamma$ by replacing at least one of the equalities $h_{j}=0$ in $\Gamma$ by either $h_{j}>0$ or $h_{j}<0$. Thus the minimal cell with respect to $\prec$ is $\Gamma_{\min }:=\left\{h_{1}=\cdots=h_{k}=\right.$ $0\}$. Clearly, the cells having the same number $p$ of equations are not pairwise comparable 
with respect to $\prec$, we say that these cells are on the level $k-p+1$. In particular, $\Gamma_{\min }$ is the only cell on level 1 .

Let

$$
1 \gg \varepsilon_{1} \gg \delta_{1} \gg \varepsilon_{2} \gg \delta_{2} \gg \cdots \gg \varepsilon_{k} \gg \delta_{k}>0,
$$

where 》stands for "sufficiently greater than". The set $X_{1}$ is the result of the following inductive construction.

Let $\Sigma_{\ell, 1}, \ldots, \Sigma_{\ell, t_{\ell}}$ be all cells on the level $\ell$ which lie in $X$. Let $\Delta_{\ell, 1}, \ldots, \Delta_{\ell, r_{\ell}}$ be all cells on the level $\ell$ which have the empty intersection with $X$. For any cell

$$
\Sigma_{\ell, j}:=\left\{h_{i_{1}}=\cdots=h_{i_{k-\ell+1}}=0, h_{i_{k-\ell+2}}>0, \ldots, h_{i_{k_{1}}}>0, h_{i_{k_{1}+1}}<0, \ldots, h_{i_{k}}<0\right\}
$$

on the level $\ell \leq k$ introduce the set

$$
\begin{aligned}
\widehat{\Sigma}_{\ell, j}:=\{ & h_{i_{1}}^{2} \leq \varepsilon_{\ell}, \ldots, h_{i_{k-\ell+1}}^{2} \leq \varepsilon_{\ell}, \\
& \left.h_{i_{k-\ell+2}} \geq 0, \ldots, h_{i_{k_{1}}} \geq 0, h_{i_{k_{1}+1}} \leq 0, \ldots, h_{i_{k}} \leq 0\right\} .
\end{aligned}
$$

Additionally, for any cell

$$
\Sigma_{k+1, j}:=\left\{h_{i_{1}}>0, \ldots, h_{i_{k_{1}}}>0, h_{i_{k_{1}+1}}<0, \ldots, h_{i_{k}}<0\right\}
$$

on the level $k+1$ let

$$
\widehat{\Sigma}_{k+1, j}:=\left\{h_{i_{1}} \geq 0, \ldots, h_{i_{k_{1}}} \geq 0, h_{i_{k_{1}+1}} \leq 0, \ldots, h_{i_{k}} \leq 0\right\} .
$$

For any cell

$$
\Delta_{\ell, j}:=\left\{h_{i_{1}}=\cdots=h_{i_{k-\ell+1}}=0, h_{i_{k-\ell+2}}>0, \ldots, h_{i_{k_{1}}}>0, h_{i_{k_{1}+1}}<0, \ldots, h_{i_{k}}<0\right\}
$$

on the level $\ell \leq k$ introduce the set

$$
\begin{aligned}
\widehat{\Delta}_{\ell, j}:= & \left\{h_{i_{1}}^{2}<\delta_{\ell}, \ldots, h_{i_{k-\ell+1}}^{2}<\delta_{\ell},\right. \\
& \left.h_{i_{k-\ell+2}}>0, \ldots, h_{i_{k_{1}}}>0, h_{i_{k_{1}+1}}<0, \ldots, h_{i_{k}}<0\right\} .
\end{aligned}
$$

Let

$$
X_{k+1}:=X \cup \bigcup_{j} \widehat{\Sigma}_{k+1, j} .
$$

Assume that $X_{\ell+1}$ is constructed. Let

$$
X_{\ell}:=\left(X_{\ell+1} \backslash \bigcup_{j} \widehat{\Delta}_{\ell, j}\right) \cup \bigcup_{j} \widehat{\Sigma}_{\ell, j} .
$$

On the last step of the induction we obtain set $X_{1}$.

Example 4 (continued). In Example 4 we have

$$
\Gamma_{\text {min }}=\Sigma_{1,1}=\Sigma_{1, t_{1}}=\left\{x^{2} y^{2}=x^{2}+y^{2}=0\right\} .
$$


Choose the following sub-indices for the non-empty cells:

$$
\begin{aligned}
& \Delta_{2,1}:=\left\{x^{2} y^{2}=0, x^{2}+y^{2}>0\right\}, \\
& \Sigma_{3,1}:=\left\{x^{2} y^{2}>0, x^{2}+y^{2}>0\right\} .
\end{aligned}
$$

Then

$$
\begin{aligned}
& \widehat{\Sigma}_{1,1}=\left\{\left(x^{2} y^{2}\right)^{2} \leq \varepsilon_{1},\left(x^{2}+y^{2}\right)^{2} \leq \varepsilon_{1}\right\}, \\
& \widehat{\Delta}_{2,1}=\left\{\left(x^{2} y^{2}\right)^{2}<\delta_{2}, x^{2}+y^{2}>0\right\}, \\
& \widehat{\Sigma}_{3,1}=\left\{x^{2} y^{2} \geq 0, x^{2}+y^{2} \geq 0\right\} .
\end{aligned}
$$

The inductive construction proceeds as follows. Since $\Sigma_{3,1}$ is the only non-empty cell on level 3, we get $X_{3}=X \cup \widehat{\Sigma}_{3,1}=X$. Next, since $\Delta_{2,1}$ is the only non-empty cell on level 2, we get $X_{2}=X_{3} \backslash \widehat{\Delta}_{2,1}$ (i.e., $X_{2}$ is $\mathbb{R}^{2}$ minus an open $\delta_{2}$-neighbourhood of the union of the coordinate axes). Finally, $X_{1}=X_{2} \cup \widehat{\Sigma}_{1,1}$, or, in terms of polynomial inequalities,

$$
X_{1}=\left(X \backslash\left\{\left(x^{2} y^{2}\right)^{2}<\delta_{2}, x^{2}+y^{2}>0\right\}\right) \cup\left\{\left(x^{2} y^{2}\right)^{2} \leq \varepsilon_{1},\left(x^{2}+y^{2}\right)^{2} \leq \varepsilon_{1}\right\} .
$$

Thus, $X_{1}$ is the plane $\mathbb{R}^{2}$ minus an open neighbourhood of the union of the coordinate axes plus a larger closed neighbourhood of the origin. Obviously, $X_{1}$ can be defined by a Boolean formula without negations, involving the same polynomials as in (2), and having only non-strict inequalities. It is easy to see that $X$ and $X_{1}$ are homotopy equivalent.

Returning to the general case, one can prove that $X$ and $X_{1}$ are weakly homotopy equivalent. For our purposes the following weaker statement will be sufficient.

Lemma 5. The sum of Betti numbers of $X$ coincides with the sum of Betti numbers of $X_{1}$.

Proof. For every $m, 1 \leq m \leq k+1$, define a set $Y^{m}$ using the inductive procedure similar to the one used for defining $X_{1}$. The difference is that the base step of the induction starts at some level $m$ rather than specifically at the level $k+1$. More precisely, let $Y^{k+1}:=X_{1}$. For any $m \leq k$, let

$$
Z_{m}^{m, 1}:=X \backslash \bigcup_{j} \widehat{\Delta}_{m, j} \quad \text { and } \quad Z_{m}^{m, 2}:=Z_{m}^{m, 1} \cup \bigcup_{j} \widehat{\Sigma}_{m, j}
$$

This concludes the base of the induction.

On the induction step, suppose that $Z_{\ell+1}^{m, s}$ is defined, where $m-1 \geq \ell \geq 1, s=1,2$. Define

$$
Z_{\ell}^{m, s}:=\left(Z_{\ell+1}^{m, s} \backslash \bigcup_{j} \widehat{\Delta}_{\ell, j}\right) \cup \bigcup_{j} \widehat{\Sigma}_{\ell, j}
$$

Let $Y^{m}:=Z_{1}^{m, 2}$.

For every $m, 1 \leq m \leq k+1$, define the set $Y^{\prime m}$ by the procedure similar to the definition of $Y^{m}$, replacing in each $\widehat{\Sigma}_{\ell, j}$ the inequalities $h_{i_{1}}^{2} \leq \varepsilon_{\ell}, \ldots, h_{i_{k-\ell+1}}^{2} \leq \varepsilon_{\ell}$ by $h_{i_{1}}^{2}<\varepsilon_{\ell}, \ldots, h_{i_{k-\ell+1}}^{2}<\varepsilon_{\ell}$, respectively, and in each $\widehat{\Delta}_{\ell, j}$ the inequalities $h_{i_{1}}^{2}<$ 
$\delta_{\ell}, \ldots, h_{i_{k-\ell+1}}^{2}<\delta_{\ell}$ by $h_{i_{1}}^{2} \leq \delta_{\ell}, \ldots, h_{i_{k-\ell+1}}^{2} \leq \delta_{\ell}$, respectively. Denote the results of the replacements by $\widehat{\Sigma}_{\ell, j}^{\prime}$ and $\widehat{\Delta}_{\ell, j}^{\prime}$, respectively.

We show by induction on $m$ that $\mathrm{b}\left(Y^{m}\right)=\mathrm{b}\left(Y^{\prime m}\right)$ and that $\mathrm{b}(X)=\mathrm{b}\left(Y^{m}\right)=\mathrm{b}\left(Y^{\prime m}\right)$. It will follow, in particular, that the sum of Betti numbers of $X$ does not exceed the sum of Betti numbers of $X_{1}=Y^{k+1}$.

For the base case of $m=1$, let first $\Gamma_{\min } \neq \emptyset$ and $\Gamma_{\min } \cap X=\emptyset$ (i.e., $\Gamma_{\min }=\Delta_{1,1}=$ $\left.\Delta_{1, r_{1}}\right)$, then

$$
Y^{1}=X \backslash \widehat{\Delta}_{1,1}=X \backslash\left\{h_{1}^{2}<\delta_{1}, \ldots, h_{k}^{2}<\delta_{1}\right\} .
$$

Introduce the following directed system of sets. First replace $\delta_{1}$ in the definition of $Y^{1}$ by a parameter and then consider the family of sets as the parameter tends to 0 . Denote this directed system by $\left\{Y^{1}\right\}_{\delta_{1} \rightarrow 0}$. Observe that $\left\{Y^{1}\right\}_{\delta_{1} \rightarrow 0}$ is a fundamental covering of $X$. Indeed, since any point $x \in X$ does not belong to the closed set $\left\{h_{1}=\cdots=h_{k}=\right.$ $0\}$, there is a neighbourhood $U$ of $x$ in $Y^{1}$ for all small enough $\delta_{1}$, which is also a neighbourhood of $x$ in $X$, such that $U \cap\left\{h_{1}=\cdots=h_{k}=0\right\}=\emptyset$. Thus, if for a subset $A \subset X$ the intersection $A \cap Y^{1}$ is open in $Y^{1}$ for any small enough $\delta_{1}$, then $A$ is open in $X$. Therefore (see Section 1.2.4.7 of [10]), $X$ is a direct limit of $\left\{Y^{1}\right\}_{\delta_{1} \rightarrow 0}$. It follows (see Theorem 4.1 .7 on p. 162 of [11]) that $H_{*}(X)$ is the direct limit of $\left\{H_{*}\left(Y^{1}\right)\right\}_{\delta_{1} \rightarrow 0}$. On the other hand, by Hardt's triviality theorem [3, p. 62, Theorem 5.22] for a small enough positive $\delta_{1}$ all $Y^{1}$ are pairwise homeomorphic. Thus, for a small enough $\delta_{1}$ we have $\mathrm{b}(X) \leq \mathrm{b}\left(Y^{1}\right)$. Moreover, we have $H_{*}(X) \simeq H_{*}\left(Y^{1}\right)$ and therefore $\mathrm{b}(X)=\mathrm{b}\left(Y^{1}\right)$. Indeed, due again to Hardt's triviality theorem, for all small enough positive values of $\delta_{1}$ the inclusion maps in the filtration of spaces $Y^{1}$ are homotopic to homeomorphisms and therefore induce isomorphisms in the corresponding direct system of groups $H_{*}\left(Y^{1}\right)$. It follows that the direct limit of groups $\left\{H_{*}\left(Y^{1}\right)\right\}_{\delta_{1} \rightarrow 0}$ is isomorphic to any of these groups for a fixed small enough positive $\delta_{1}$.

Observe that a similar argument is applicable to $Y^{\prime 1}=X \backslash\left\{h_{1}^{2} \leq \delta_{1}, \ldots, h_{k}^{2} \leq \delta_{1}\right\}$, therefore $H_{*}(X) \simeq H_{*}\left(Y^{\prime 1}\right)$.

Suppose now that $\Gamma_{\min } \neq \emptyset$ and $\Gamma_{\min } \subset X$ (i.e., $\Gamma_{\min }=\Sigma_{1,1}=\Sigma_{1, t_{1}}$ ). Then $\Gamma_{\min } \cap \widetilde{X}=\emptyset$, where $\widetilde{X}$ is the complement of $X$. Replacing in the above proof the set $X$ by $\widetilde{X}$, and $\delta_{1}$ by $\varepsilon_{1}$, we get $H_{*}(\widetilde{X}) \simeq H_{*}\left(\widetilde{Y^{\prime 1}}\right)$. Since $X$ is bounded, by Alexander's duality, $\mathrm{b}(\widetilde{X})=\mathrm{b}(X)+1$ and $\mathrm{b}\left(Y^{\prime 1}\right)=\mathrm{b}\left(Y^{\prime 1}\right)+1$, hence $\mathrm{b}(X)=\mathrm{b}\left(Y^{\prime 1}\right)$.

Similar argument shows that $\mathrm{b}(X)=\mathrm{b}\left(Y^{1}\right)$.

The case when $\Gamma_{\min }=\emptyset$ is trivial. This concludes the base induction step.

Assume that $\mathrm{b}(X)=\mathrm{b}\left(Y^{m}\right)=\mathrm{b}\left(Y^{\prime m}\right)$. First let $\bigcup_{j} \Delta_{m+1, j} \neq \emptyset$, then the family of sets $\left\{Z_{1}^{\prime m+1,1}\right\}_{\delta_{m+1} \rightarrow 0}$ is a fundamental covering of $Y^{\prime m}$. Indeed, by the definition we have

$$
Z_{m+1}^{\prime m+1,1}=X \backslash \bigcup_{j} \widehat{\Delta}_{m+1, j}^{\prime}
$$

Take any point $x \in Z_{1}^{\prime m+1,1}$. Then $x$ belongs either to

$$
\bigcap_{j}\left(\left\{h_{i_{1}}^{2}>\delta_{m+1}\right\} \cup \cdots \cup\left\{h_{i_{k-m}}^{2}>\delta_{m+1}\right\}\right)
$$

for all non-empty cells

$$
\Delta_{m+1, j}=\left\{h_{i_{1}}=\cdots=h_{i_{k-m}}=0, h_{i_{k-m+1}}>0, \ldots, h_{i_{k}}<0\right\}
$$


and all sufficiently small $\delta_{m+1}$, or to a set of the kind

$$
\begin{gathered}
\left\{h_{i_{1}}=\cdots=h_{i_{k-m}}=0, h_{i_{k-m+1}}^{2}<\varepsilon_{t}, \ldots, h_{i_{k-t+1}}^{2}<\varepsilon_{t},\right. \\
\left.h_{i_{k-t+2}}>0, \ldots, h_{i_{k_{1}}}>0, h_{i_{k_{1}+1}}<0, \ldots, h_{i_{k}}<0\right\}
\end{gathered}
$$

for some $t \leq m$ and a non-empty cell

$$
\Sigma_{t, j}=\left\{h_{i_{1}}=\cdots=h_{i_{k-t+1}}=0, h_{i_{k-t+2}}>0, \ldots, h_{i_{k}}<0\right\} \subset X .
$$

In both cases there is a set $U$ which is a neighbourhood of $x$ in $Z_{1}^{\prime m+1,1}$ for all sufficiently small $\delta_{m+1}$, and also a neighbourhood of $x$ in $Y^{\prime m}$.

Thus, for a small enough $\delta_{m+1}$ we have $H_{*}\left(Y^{\prime m}\right) \simeq H_{*}\left(Z_{1}^{\prime m+1,1}\right)$. Introduce a set $Z_{1}^{\prime m+1,1}(\gamma)$, where $0<\gamma \ll \delta_{m+1}$, defined by a formula $\varphi(\gamma)$ which is constructed as follows. In the formula $\varphi$ defining $Z_{1}^{\prime m+1,1}$ replace all occurrences of the systems of inequalities of the kind $h_{i_{1}}^{2}<\varepsilon_{\ell}, \ldots, h_{i_{k-\ell+1}}^{2}<\varepsilon_{\ell}$ by $h_{i_{1}}^{2} \leq \varepsilon_{\ell}-\gamma, \ldots, h_{i_{k-\ell+1}}^{2} \leq \varepsilon_{\ell}-\gamma$ and all occurrences of the systems inequalities of the kind $h_{i_{1}}^{2} \leq \delta_{\ell}, \ldots, h_{i_{k-\ell+1}}^{2} \leq \delta_{\ell}$ by $h_{i_{1}}^{2}<\delta_{\ell}+\gamma, \ldots, h_{i_{k-\ell+1}}^{2}<\delta_{\ell}+\gamma$. The family of sets $\left\{Z_{1}^{\prime m+1,1}(\gamma)\right\}_{\gamma \rightarrow 0}$ is a fundamental covering of $Z_{1}^{\prime m+1,1}$, thus for a small enough $\gamma$ we have

$$
H_{*}\left(Z_{1}^{\prime m+1,1}\right) \simeq H_{*}\left(Z_{1}^{\prime m+1,1}(\gamma)\right)
$$

However, the sets $Z_{1}^{\prime m+1,1}(\gamma)$ and $Z_{1}^{m+1,1}$ are homeomorphic due to Hardt's triviality theorem, therefore $H_{*}\left(Z_{1}^{\prime m+1,1}\right) \simeq H_{*}\left(Z_{1}^{m+1,1}\right)$. It follows that

$$
\mathrm{b}(X)=\mathrm{b}\left(Y^{\prime m}\right)=\mathrm{b}\left(Z_{1}^{\prime m+1,1}\right)=\mathrm{b}\left(Z_{1}^{m+1,1}\right) .
$$

Now let $\bigcup_{j} \Sigma_{m+1, j} \neq \emptyset$. Note that $\tilde{X} \cap \bigcup_{j} \Sigma_{m+1, j}=\emptyset$. As above (but using $\varepsilon_{m+1}$ in place of $\delta_{m+1}$ ), we get

$$
\mathrm{b}(\widetilde{X})=\mathrm{b}\left(\widetilde{Z}_{1}^{m+1,2}\right)=\mathrm{b}\left(\widetilde{Z}_{1}^{\prime m+1,2}\right)
$$

By Alexander's duality we have $\mathrm{b}(\tilde{X})=\mathrm{b}(X)+1, \mathrm{~b}\left(\widetilde{Z}_{1}^{m+1,2}\right)=\mathrm{b}\left(Z_{1}^{m+1,2}\right)+1$, and $\mathrm{b}\left(\widetilde{Z}_{1}^{\prime m+1,2}\right)=\mathrm{b}\left(Z_{1}^{\prime m+1,2}\right)+1$, hence in this case the condition

$$
\mathrm{b}(X)=\mathrm{b}\left(Z_{1}^{m+1,2}\right)=\mathrm{b}\left(Z_{1}^{\prime m+1,2}\right)
$$

is also true.

The case when $\bigcup_{j}\left(\Delta_{m+1, j} \cup \Sigma_{m+1, j}\right)=\emptyset$ is trivial.

Recalling that $Z_{1}^{m+1,2}=Y^{m+1}$ and $Z_{1}^{\prime m+1,2}=Y^{\prime m+1}$, we get the required $\mathrm{b}(X)=$ $\mathrm{b}\left(Y^{m+1}\right)=\mathrm{b}\left(Y^{\prime m+1}\right)$.

Proof of Theorem 1. According to Lemma 5, it is sufficient to prove the bound for the set $X_{1}$ which is defined by a Boolean combination (with no negations) of non-strict inequalities. The atomic polynomials are either of the kind $h_{i}$ or of the kind $h_{i}^{2}-\delta_{j}$ or of the kind $h_{i}^{2}-\varepsilon_{j}, 1 \leq i, j \leq k$, hence there is at most $O\left(k^{2}\right)$ pairwise distinct among them. Now the theorem follows from Proposition 2. 
Remark 6. Employing some additional technicalities one can prove that in the construction of set $X_{1}$ it is sufficient to use just one sort of constants, i.e., keep $\varepsilon_{1}, \ldots, \varepsilon_{k}$ in their positions and replace $\delta_{1}, \ldots, \delta_{k}$ by $\varepsilon_{1}, \ldots, \varepsilon_{k}$, respectively. This reduces the number of polynomials involved in the description of $X_{1}$ and therefore the $O$-symbol constant in the upper bound of Theorem 1.

\section{Acknowledgements}

The authors thank S. Basu for useful discussions.

\section{References}

1. S. Basu, On bounding the Betti numbers and computing the Euler characteristic of semi-algebraic sets, Discrete Comput. Geom. 22 (1999), 1-18.

2. S. Basu, R. Pollack, and M.-F. Roy, Algorithms in Real Algebraic Geometry, Springer-Verlag, Berlin, 2003.

3. M. Coste, An Introduction to o-Minimal Geometry, Dip. Mat. Univ. Pisa, Dottorato di Ricerca in Matematica, Istituti Editoriale e Poligrafici Internazionali, Pisa, 2000.

4. D. Grigoriev, Complexity of deciding Tarski algebra, J. Symbolic Comput. 5 (1988), 65-108.

5. J. Heintz, Definability and fast quantifier elimination in algebraically closed fields, Theoret. Comput. Sci. 24 (1983), 239-277.

6. J. Milnor, On the Betti numbers of real varieties, Proc. Amer. Math. Soc. 15 (1964), 275-280.

7. J. L. Montaña, J. E. Morais, and L. M. Pardo, Lower bounds for arithmetic networks, II: sum of Betti numbers, Appl. Algebra Engrg. Comm. Comput. 7 (1996), 41-51.

8. O. A. Oleinik, Estimates of the Betti numbers of real algebraic hypersurfaces (Russian), Mat. Sb. 28 (1951), 635-640.

9. O. A. Oleinik and I. G. Petrovskii, On the topology of real algebraic hypersurfaces (Russian), Izv. Acad. Nauk SSSR 13 (1949), 389-402. English transl.: Amer. Math. Soc. Transl. 7 (1962) 399-417.

10. V. A. Rokhlin and D. B. Fuks, Beginners Course in Topology. Geometric Chapters, Springer-Verlag, New York, 1984

11. E. Spanier, Algebraic Topology, Springer-Verlag, New York, 1981.

12. R. Thom, Sur l'homologie des variétés algebriques réelles, in: S. S. Cairns (ed.), Differential and Combinatorial Topology, pp. 255-265, Princeton University Press, Princeton, NJ, 1965.

13. A. C. C. Yao, Decision tree complexity and Betti numbers, in: Proc. of 26th ACM Symp. on Theory of Computing, Montreal, Canada, pp. 615-624, ACM Press, New York, 1994.

Received July 24, 2003, and in revised form January 15, 2004. Online publication May 28, 2004. 